\title{
Mayor actividad de rho kinasa en leucocitos circulantes se asocia a estrés oxidativo y rigidez arterial en hipertensos diabéticos
}

\author{
Luigi Gabrielli, Alejandro Berkovitz, Italo Mora, Ulises Novoa, Iván Godoy, Paul MacNab, Samuel Córdova, \\ Ivonne Padilla, Paola Rigotti, Lorena García, Sergio Lavandero, María Paz Ocaranza, Jorge Jalil.
}

División de Enfermedades Cardiovasculares. Pontificia Universidad Católica de Chile.

\section{Resumen:}

Introducción: La vía intracelular de RhoA/Rho kinasa es activada por agonistas de receptores acoplados a proteínas $\mathrm{G}$ pequeñas unidas a membrana. Su activación está relacionada al remodelado cardiovascular patológico. Previamente hemos observado aumento de actividad de Rho kinasa (ROCK) en pacientes con hipertensión arterial (HT) e hipertrofia ventricular izquierda como daño de órgano blanco. Pero su activación en relación a la diabetes no ha sido explorada en estos pacientes.

Objetivo: Evaluar activación de Rho kinasa y parámetros de estrés oxidativo en pacientes hipertensos con diabetes tipo II (DMII).

Métodos: Estudio comparativo entre pacientes con HT sin tratamiento, HT con DMII y hemoglobina glicosilada Alc $\geq 7,5 \%$ y un grupo control normotenso. Se realizó ecocardiograma de superficie. Se midió activación de ROCK en leucocitos circulantes midiendo MYPT1 fosforilado/total $(\mathrm{p} / \mathrm{t})$ por Western blot y la velocidad de pulso carotídeo-femoral (PWV) para estimar distensibili- dad arterial. El stress oxidativo se estimó midiendo malondialdehído (MDA) y 8-isoprostano (8-ISO) en suero.

Resultados: Se incluyeron 21 pacientes hipertensos con DMII, 38 pacientes hipertensos sin DMII y 34 controles normotensos. La edad promedio fue $51 \pm 0,9 ; 48 \pm$ 0,9 y 52 (p: NS) $\pm 1,1$ y el $47 \%, 50 \%$ y $52 \%$ (p: NS) eran mujeres respectivamente. Los pacientes HT con DMII presentaron MYPT1 p/t $(5,6 \pm 1,3 ; 3,6 \pm 0,4 ; 2,1 \pm 0,1$ $\mathrm{p}<0,01), \operatorname{MDA}(1,8 \pm 0,4 \mu \mathrm{M} ; 0,49 \pm 0,08 ; 0,4 \pm 0,06 \mathrm{p}<$ 0,01), 8-ISO (C) y PWV (14 $\pm 0,9$ m/seg; $11 \pm 0,4 ; 8,1 \pm$ $0,1)$ elevados versus los otros grupos.

Conclusiones: Los pacientes hipertensos con DM II presentan actividad de ROCK elevada respecto a hipertensos sin DM II a igual IMVI, además presentan parámetros de estrés oxidativo aumentados respecto a HT sin DM II y controles normotensos. Estos hallazgos están relacionados con una distensibilidad arterial alterada y podrían explicar en parte el mayor remodelado vascular patológico que presentan los pacientes HT con DM II.

Correspondencia:

Jorge Jalil Milad

Jefe División de Enfermedades Cardiovasculares

Universidad Católica

jjalil@med.puc.cl

Luigi Gabrielli

Instructor Asociado División de Enfermedades

Cardiovasculares UC

Igabriel@uc.cl 
Background: Rho/Rho-kinase intracellular pathway is activated by membrane bound small G-proteins. Activation of Rho/Rho-kinase pathway is related to pathologic cardiac remodeling. We have previously observed

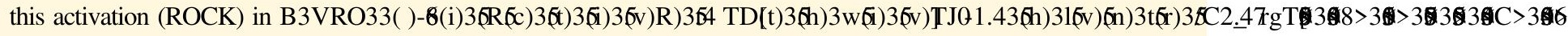


distensibilidad arterial en pacientes hipertensos esenciales (HT) con DMII.

\section{Métodos}

Estudio transversal en el cual se incluyeron tres grupos, uno formado por pacientes HT recientemente diagnosticados sin terapia, un grupo de HT con DMII cuya hemoglobina glicosilada A1C al momento del ingreso al estudio fuera $\geq 7,5 \%$ con tratamiento hipotensor e hipoglicemiante y un grupo control normotenso sin DMII pareado por edad y sexo. Los criterios de exclusión fueron: edad $<18$ años, insuficiencia renal (creatinina $\geq 1,5 \mathrm{mg} / \mathrm{dL}$ ), enfermedad hepática (transaminasa oxaloacética $>1,5$ veces el valor normal alto), neoplasia en los últimos 4 años, infección activa (PCR > $1 \mathrm{mg} / \mathrm{dL}$ ), usuarios de insulina, usuario de estatinas, historia de insuficiencia cardíaca o disfunción ventricular (fracción de eyección $<50 \%$ ), antecedente de enfermedad coronaria y enfermedad pulmonar obstructiva crónica.

Todos los sujetos del estudio firmaron un consentimiento informado aprobado por un comité de ética local.

Se registraron la características demográficas y parámetros de laboratorio clínico en todos los sujetos. Se midieron hemoglobina glicosilada A1C, glicemia, creatinina plasmática, niveles de colesterol total y LDL, ácido úrico y transaminasa oxaloacética.

\section{Medición de la presión arterial}

En todos los sujetos se evaluó la presión arterial sistólica y diastólica (en posición sentado) utilizando un esfingomanómetro de mercurio estándar. La fase I y V de los sonidos de Korotkoff se utilizaron para este fin. Se realizaron 3 determinaciones en dos días separados, a intervalos de 1 minuto y luego de 5 minutos de reposo. El diagnóstico de HT se consideró con valores > 140/90 de presión sistólica o diastólica respectivamente. Se consideró normotensión cuando ambos valores estuvieron bajo los mencionados anteriormente.

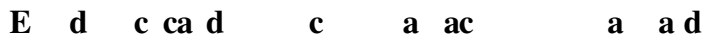 la rigidez arterial.}

En todos los sujetos se realizó un ecocardiograma de superficie en decúbito lateral izquierdo obteniéndose las visiones paraesternales y apicales habituales. Se utilizó un equipo Phillips IE-33 con un transductor S5-1 de 2,5-5
$\mathrm{MHz}$ por un operador experto y ciego. Se evaluó la función y masa ventricular izquierda, el tamaño auricular izquierdo (AI) y parámetros de función distólica. Todas las mediciones se hicieron según las recomendaciones de la American Society of Echocardiography ${ }^{16,17}$. Para el cálculo de la masa ventricular izquierda se utilizó la fórmula de Devereux ${ }^{18,19}$ y los valores fueron indexados. Como método de evaluación de la función sistólica se utilizó el cálculo de la fracción de eyección ventricular izquierda (FEVI) por el método de Simpson. Además, se determinó la velocidad de pulso carótido-femoral (PWV) como índice indirecto y no invasivo de la rigidez arterial. Para este fin se utilizó un equipo Complior $1^{20}$.

\section{Pa d da}

En todos los sujetos se midieron los niveles plasmáticos de malondialdehído (MDA) y de 8-Isoprostano (8-iso) como parámetros de evaluación del estrés oxidativo. Se obtuvieron $10 \mathrm{~mL}$ de sangre venosa periférica. La muestra fue centrifugada a $3000 \mathrm{rpm}$ por 10 minutos a una temperatura de $4{ }^{\circ} \mathrm{C}$. El plasma fue separado y almacenado a $-20{ }^{\circ} \mathrm{C}$. Los niveles de MDA fueron determinados por el contenido de substancias reactivas al ácido tiobarbitúrico

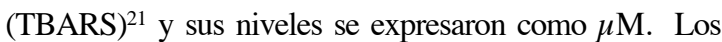
niveles de 8-ISO fueron evaluados mediante un kit de inmunoensayo enzimático disponible comercialmente (Cayman Chem Co., Ann Arbor, MI) y sus niveles se expresaron como $\mathrm{pg} / \mathrm{mL}^{21}$.

\section{M d c d ac $\operatorname{dad} d \mathrm{R}-\mathbf{a} \mathbf{a}$}

La actividad de ROCK se evaluó midiendo la relación entre la fosfatasa de la cadena liviana de la miosina 1 fosforilada versus la total (MYPT1-p/t) en leucocitos circulantes, un objetivo directo de la vía de ROCK. Diez mililitros de sangre venosa con EDTA fueron vertidos sobre 10 mililitros de Histopaque (Histopaque-1077, Sigma Chemical Co., St. Louis, Missouri) y luego centrifugados. Los leucocitos fueron resuspendidos en PBS y centrifugados a temperatura ambiente dos veces. Se determinó la viabilidad celular mediante el test de exclusión de azul de tripan (usualmente 4 a 80 X 106 células viables con una viabilidad mayor del $95 \%$ ) y luego se resuspendieron en un buffer de lisis. Las muestras fueron centrifugadas y el contenido proteico del sobrenadante fue determinado usando el ensayo de Bradford empleando BSA como estándar. La fracción soluble fue calentada a $95^{\circ} \mathrm{C}$ con muestra buffer SDS-PAGE para MYPT-1 para el análisis por Western blot ${ }^{22}$. 


\section{Análisis estadístico}

Para el cálculo del tamaño muestral se utilizó el programa Power and Sample size calculations versión 2.1.31 . Para una diferencia esperada de $20 \%$, una potencia de $90 \%$, error alfa de 5\% los grupos debían ser al menos de 15 sujetos. Las variables discretas de expresaron como porcentajes y las continuas como promedios \pm error estándar (EE). Para evaluar las diferencias entre los grupos se utilizó ANOVA de una cola y test post hoc de Bonferroni. Para evaluar correlaciones se utilizó el método de Pearson.

\section{Resultados}

Se incluyeron 21 pacientes HT con DMII con tratamiento 


\section{E d d da}

Los pacientes HT con DMII presentaron niveles elevados de MDA y de 8-ISO respecto de los HT sin DMII y controles. Los pacientes HT sin DMII y controles presentaron niveles similares de ambos marcadores de estrés oxidativo. (Figuras 2 y 3 ).

Figura 2: MDA plasm tico en grupos control, HT sin DMII e HT con DMII.
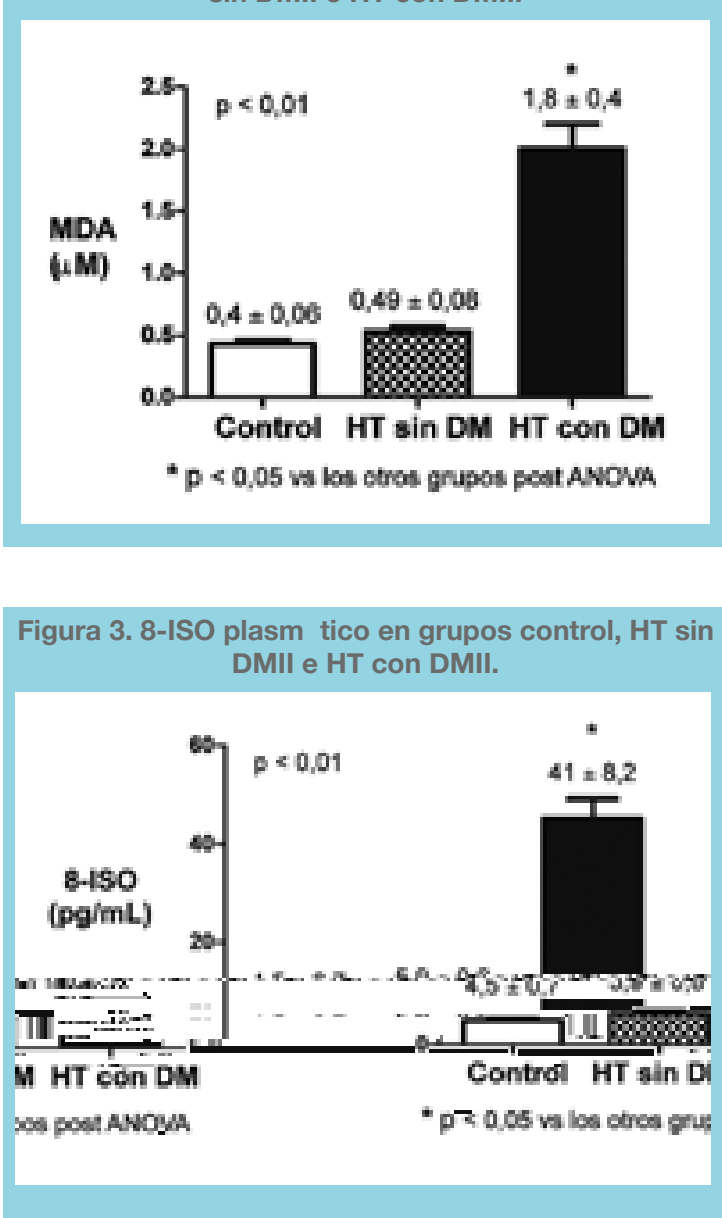

\section{Ac $\operatorname{dad} \mathbf{d} \mathbf{R}$ a a}

Los pacientes HT con DMII presentaron actividad de ROCK significativamente elevada respecto a HT sin DMII y controles. Entre los pacientes HT sin DMII y el grupo control la diferencia observada no alcanzó significancia estadística $(\mathrm{p}=0.09)$ (Figura 4).

No se encontraron correlaciones significativas entre los niveles de ROCK, estudio de rigidez arterial y parámetros de estrés oxidativo evaluados en los pacientes HT como grupo total y en los con y sin DMII en forma aislada.
Figura 4. MYPT-1 p/t en leucocitos circulantes en grupos control, HT sin DMII e HT con DMII. (p/t: fosforilado versus total).

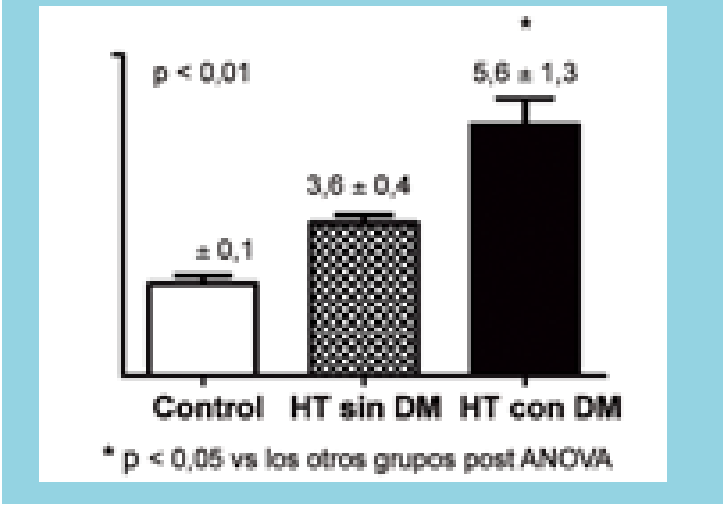

\section{Discusión}

Los principales hallazgos del presente estudio son que los pacientes HT con DM II presentan actividad de ROCK elevada respecto a hipertensos sin DM II a igual IMVI, además, presentan parámetros de estrés oxidativo aumentados respecto a HT sin DM II y controles normotensos. Estos hallazgos están relacionados con una distensibilidad arterial alterada y podrían explicar en parte, las alteraciones vasculares de los pacientes HT con DM II. Desde el punto de vista clínico sólo existía evidencia de aumento de actividad de ROCK en leucocitos circulantes de pacientes con síndrome metabólico ${ }^{22}$, sin haberse descrito estos hallazgos en la población de este estudio.

En modelos experimentales se ha demostrado que un aumento de la actividad de ROCK, por estimulación del receptor $\beta$ del factor de crecimiento derivado de plaquetas, está ligada a migración y proliferación de células musculares lisas una característica principal del daño macroangiopático por diabetes ${ }^{23}$. La vía RhoA/Rho kinasa está involucrada en el aumento de actividad del factor de crecimiento tumoral $\beta$ que produce aumento de matriz extracelular y fibrosis en glomérulos de ratas diabéticas ${ }^{24}$ y que el daño de asociado a la nefropatía diabética es revertido por Fasudil, un antagonista de la vía RhoA/Rho kinasa ${ }^{25}$. Además se ha visto que MYPT1 fosforilado se encuentra aumentado en la retina de ratas diabética luego de 2 semanas del inicio de la diabetes ${ }^{26} \mathrm{y}$ que la terapia con estatinas disminuye la actividad de ROCK y esto se asocia a disminución del daño cicatricial del vítreo y retina de ratas con retinopatía diabética ${ }^{27}$. Toda esta evidencia está en concordancia con el hallazgo clínico de nuestro estudio, de un claro aumento de la actividad de ROCK en pacientes HT con DMII independiente del IMVI y que probablemente está involucrada 
en las complicaciones micro y macro angiopáticas de la misma.

En los pacientes HT con DMII se observa una rigidez arterial aumentada, hecho clínico antes descrito ${ }^{28,29}$, lo cual en nuestros pacientes está asociado a una actividad de ROCK elevada. Sin embargo, no encontramos una correlación significativa entre estos hallazgos probablemente porque en el daño vascular del paciente diabético están involucrados muchos otros factores y uno en particular no es capaz de explicar el fenómeno.

Otro de los factores involucrados en el daño vascular del paciente HT con DMII es el estrés oxidativo. En pacientes diabéticos, la hiperglicemia produce un aumento de la producción de especies reactivas de oxígeno a través de un incremento de la actividad de NADPH oxidasa ${ }^{30}$, esto está ligado a disfunción endotelial, menor disponibilidad de óxido nítrico ${ }^{31}$ y es considerado clave en la génesis de la nefropatía diabética ${ }^{32}$. Por otro lado, se ha mostrado en modelos de isquemia reperfusión que un aumento en la actividad de la vía RhoA/Rho kinasa está ligada a mayor producción de especies reactivas de oxígeno vía NADPH oxidasa 1 y a disfunción endotelial ${ }^{33}$. En nuestro estudio observamos un aumento en los marcadores de estrés oxidativo, MDA y 8-ISO, en los pacientes HT con DMII, este aumento podría estar dado en parte, por el aumento de la actividad de la vía RhoA/Rho kinasa demostrada en estos pacientes. No encontramos correlación significativa entre los marcadores de estrés oxidativo empleados y los niveles de actividad de ROCK, esto último se explica por la gran cantidad de sistemas involucrados en la generación de especies reactivas de oxígeno y que contribuyen a las alteraciones vasculares en estos pacientes ${ }^{34}$.

La diferencia encontrada en la actividad de ROCK entre hipertensos diabéticos y no diabéticos en nuestro estudio podría estar incluso subestimada, ya que los pacientes diabéticos se encontraban en su totalidad con antagonistas del sistema renina-angiotensina-aldosterona cuya actividad está ligada al sistema RhoA/Rho kinasa ${ }^{35}$ y su antagonismo está asociado a una disminución de la actividad de ROCK y del remodelado vascular patológico ${ }^{36}$. Respecto a esto último, queda abierta la pregunta clínica sobre el comportamiento de la actividad del sistema RhoA/ Rho kinasa en pacientes hipertensos diabéticos luego de un control óptimo de sus cifras tensionales y parámetros metabólicos.

Por tanto concluimos que pacientes HT con DMII presentan actividad de ROCK elevada. Esta vía está involucrada en múltiples mecanismos fisiopatológicos como estrés oxidativo, vasoconstricción y proliferación celular que pueden explicar, en parte, el daño micro y macro vascular, y podría constituir un objetivo terapéutico importante en estos pacientes.

\section{Referencias:}

1. JALIL JE, LAVANDERO S, CHIONG M, OCARANZA MP. Rho/Rho-kinase signal transduction pathway in cardiovascular disease and cardiovascular remodeling. Rev Esp Cardiol. 2005; 58:951-961.

2. SHIMOKAWA H, TAKESHITA A. Rho-kinase is an important therapeutic target in cardiovascular medicine. Arterioscler Thromb Vasc Biol. 2005;25:1767-1775.

3. Shimokawa H, Rashid M. Development of Rho-kinase inhibitors for cardiovascular medicine. Trends in Pharm Sciences 2007; 28:296-302.

4. HIGASHI M, SHIMOKAWA H, HATTORI T, HIROKI J, MUKAI Y, MORIKAWA K, et al. Long term inhibition of Rho-kinase suppresses angiotensin II-induced cardiovascular hypertrophy in rats in vivo: effect on endothelial NAD(P)H oxidase system. Circ Res 2003; 93:767-775.

5. ZHOU Q, LIAIO JK. Rho kinase: an important mediator of atherosclerosis and vascular disease. Curr Pharm Des 2009; 15:3108-3115.

6. LUIGI GABRIELLI, JORGE JALIL, IVÁN GODOY, PAUL MCNAB, IVONNE PADILLA, SAMUEL CORDOVA. Rho-kinase activity is increased in hypertensive patients with left ventricular hypertrophy. JACC 2010; 55:A60.E577.

7. MITA S, KOBAYASHI N, YOSHIDA K, NAKANO S, MATSUOKA H. Cardioprotective mechanisms of Rho-kinase inhibition associated with eNOS and oxydative stressLOX-1 pathway in Dahl salt-sensitive hypertensive rats. J Hypertens. 2005; 23:87-96.

8. MIYATA K, SHIMOKAWA H, KANDABASHI T, HIGO T, MORISHIGE K, ETO Y, et al. Rho kinase is involved in macrophage-mediated formation of coronary vascular lesions in pigs in vivo. Arterioscler Thromb Vasc Biol 2000; 20:2351-2358.

9. PEDROSA K, RIGSBY C, CLINTON R. RhoA/Rho kina- 
se and vascular diseases: what is the link?. Cell Mol Life Sci 2010; 67:3823-3826.

10. OKSON EB, SZADO T, LAHER I, MCMANUS B. Augmented contractile response of vascular smooth muscle in a diabetic mouse model. J Vasc Res 2003; 40:520-530.

11. REN J, DUAN J, THOMAS DP, YANG X, SREEJAYAN N, SOWERS JR. IGF-1 alleviates diabetes induced RhoA activation, Enos uncoupling and myocardial dysfunction. Am J Physiol Regul Integr Comp Physiol 2008; 294:R793802.

12. HIROSE A, TANIKAWA T, MORI H, OKADA Y, TANAKA Y. Advanced glycation end products increase endothelial permeability through the RAGE/Rho signaling pathway. FEBS Lett 2010; 584:61-66.

13. ZHOU HONG, LI YONG-JUN. RhoA/Rho kinase: a novel therapeutic target in diabetic complications. Chinese Medical Journal 2010; 123:2461-2466.

14. PENG F, WU D, GAO B, INGRAM AJ, ZHANG B, CHORNEYKO K, et al. RhoA/Rho kinase contribute to the pathogenesis of diabetic renal disease. Diabetes 2008; 57: 1683-1692.

15. KOMERS R, OYAMA TT, BEARD DR, TIKELLIS C, XU $\mathrm{B}$, LOTSPEICH DF, et al. Rho kinase inhibition protects kidneys from diabetic nephropathy without reducing blood pressure. Kidney Int. 2011; 79: 432-42.

16. REEVES ST, GLAS KE, ELTZSCHIG H, MATHEW JP, RUBENSON DS, HARTMAN GS, et al. American Society of Echocardiography; Society of Cardiovascular Anesthesiologists. Guidelines for performing a comprehensive epicardial echocardiography examination: recommendations of the American Society of Echocardiography and the Society of Cardiovascular Anesthesiologists. J Am Soc Echocardiogr. 2007; 20: 427-437.

17. LANG RM, BIERIG M, DEVEREUX RB, FLACHSKAMPF FA, FOSTER E, PELLIKKA PA, et al. Chamber Quantification Writing Group; American Society of Echocardiography's Guidelines and Standards Committee; European Association of Echocardiography. Recommendations for chamber quantification: a report from the American Society of Echocardiography's Guidelines and Standards Committee and the Chamber Quantification Writing Group, developed in conjunction with the European Association of Echocardiography, a branch of the European Society of Cardiology. J Am Soc Echocardiogr. 2005; 18:1440-1463.

18. DEVEREUX RB, ALONSO DR, LUTAS EM, GOTTLIEB GJ, CAMPO E, SACHS I, et al. Echocardiographic assessment of left ventricular hypertrophy: comparison to necropsy findings. Am J Cardiol. 1986;57:450-458.
19. PALMIERI V, DAHLÖF B, DEQUATTRO V, SHARPE N, BELLA JN, DE SIMONE G, et al. Reliability of echocardiographic assessment of left ventricular structure and function: the PRESERVE study. Prospective Randomized Study Evaluating Regression of Ventricular Enlargement. J Am Coll Cardiol. 1999; 34:1625-1632.

20. ASMAR R, TOPOUCHIAN J, PANNIER B, BENETOS A, SAFAR M. Scientific, Quality Control, Coordination and Investigation Committees of the Complior Study. Pulse wave velocity as endpoint in large-scale intervention trial. The Complior study. Scientific, Quality Control, Coordination and Investigation Committees of the Complior Study. J Hypertens. 2001; 19:813-818.

21. PÉREZ O, CASTRO P, DÍAZ-ARAYA G, NETTLE D, MORAGA F, CHIONG M, et al. Persistence of oxydative stress after heart transplantation: a comparative study of patients with heart transplant versus chronic stable heart failure Rev Esp Cardiol. 2002; 55:831-837.

22. LIU PY, CHEN JH, LIN LJ, LIAO JK. Increased Rhokinase activity in a Taiwanese population with metabolic syndrome. J Am Coll Cardiol. 2007;49:1619-1624.

23. AKIYAMA N, NARUSE K, KOBAYASHI Y, NAKAMURA N, HAMADA Y, NAKASHIMA E, et al. High glucose-induced upregulation of RhoA/Rho kinase via platelet-derived growth factor receptor- $\beta$ increases migration of aortic smooth muscle cells. J Mol Cell Cardiol 2008;45:326-332.

24. KOLAVENNU V, ZENG LX, PENG H, WANG Y, DANESH FR. Targeting of RhoA/Rho kinase signaling ameliorates progression of diabetic nephropathy independent of glucose control. Diabetes 2008; 57:714-723.

25. GOJO A, UTSUNOMIYA K, TANIGUCHI K, YOKOTA T, ISHIZAWA S, KANAZAWA Y, et al. The Rho-kinase inhibitor, fasudil, attenuates diabetic nephropathy in streptozotocin-induced diabetic rats. Eur J Pharmacol 2007; 568:242-247.

26. ARITA R, HATA Y, NAKAO S, KITA T, MIURA M, KAWAHARA $S$, et al. Rho kinase inhibition by fasudil ameliorates diabetes-induced microvascular damage. Diabetes 2009; 58:215-226.

27. KAWAHARA S, HATA Y, KITA T, ARITA T, MIURA M, NAKAO S, et al. Potent inhibition of cicatricial contraction in proliferative vitreoretinal diseases by statins. Diabetes 2008; 57:2784-2793.

28. JADHAV UM, KADAM NN. Non-invasive assessment of arterial stiffness by pulse-wave velocity correlates with endothelial dysfunction. Indian Heart J. 2005; 57:226-32.

29. HENRY RM, KOSTENSE PJ, SPIJKERMAN AM, DEKKER JM, NIJPELS G, HEINE RJ, et al. Arterial stiffness 
Mayor actividad de rho kinasa en leucocitos circulantes se asocia a estr s oxidativo y rigidez arterial en hipertensos diab ticos Gabrielli L, et al.

increases with deteriorating glucose tolerance status: the Hoorn Study. Circulation. 2003; 107:2089-95.

30. GAO L, MANN GE. Vascular NAD(P)H oxidase activation in diabetes: a double-edged sword in redox signalling. Cardiovasc Res. 2009; 82:9-20.

31. TABIT CE, CHUNG WB, HAMBURG NM, VITA JA. Endothelial dysfunction in diabetes mellitus: molecular mechanisms and clinical implications. Rev Endocr Metab Disord. 2010; 11:61-74.

32. FORBES JM, COUGHLAN MT, COOPER ME. Oxidative stress as a major culprit in kidney disease in diabetes. Diabetes. 2008; 57 :1446-54.

33. SHIOTANI S, SHIMADA M, TAKETOMI A, SOEJIMA Y, YOSHIZUMI T, HASHIMOTO K, et al. Rho-kinase as a novel gene therapeutic target in treatment of cold ische- mia/reperfusion-induced acute lethal liver injury: effect on hepatocellular NADPH oxidase system. Gene Ther. 2007; 14:1425-33.

34. ENDEMANN DH, SCHIFFRIN EL. Nitric oxide, oxidative excess, and vascular complications of diabetes mellitus. Curr Hypertens Rep. 2004; 6 :85-9.

35. RIVERA P, OCARANZA MP, LAVANDERO S, JALIL J. Rho kinase activation and gene expression related to vascular remodeling in normotensive rats with high angiotensin I converting enzyme levels. Hypertension 2007; 50:792-798.

36. JALIL JE, JALIL R, OCARANZA MP. Combined rho kinase and renin-angiotensin system inhibition: a new therapeutic perspective for renal and cardiovascular remodeling. Hypertens Res. 2010; 33 :883-5. 